\title{
The storage of physical and name properties in short-term visual memory
}

\author{
ROBERT A. REEVE \\ University of Sydney, Sidney. New South Wales, Australia 2006 \\ and \\ RALPH HALL \\ Lniversity of New South W'ales. Kensington. Australia 2033
}

\begin{abstract}
Two experiments investigated the hypothesis that short-term visual memory is based primarily on physical features of the visual input. Subjects were required to recall visually presented figures or the names of those figures presented either visually or aurally at a number of different retention intervals. Subjects shadowed words during the retention interval presented aurally in Experiment I and visually in Experiment II. In both experiments, figures were recalled better than names and no differences in recall of names were found due to presentation modality. Recall of both names conditions showed a steady decline across retention intervals whereas recall of figures remained at a relatively high level. These findings were interpreted as providing further support for the existence of short-term visual memory not subject to auditory recoding and based primarily on physical features of the input. It was suggested that such visual memory is limited in capacity so that input exceeding this capacity is recoded into an auditory-verbal-linguistic form.
\end{abstract}

1 The persistence of short-term memory for visual information has been demonstrated in a number of studies (e.g., Kroll. 1972: Kroll, Parks, Parkinson, Bieber. \& Johnson, 1970; Parkinson, 1972; Parkinson, Parks, \& Kroll, 1971). Evidence that the storage is of a visual nature was provided by the finding that a single letter (Kroll et al., 1970), and four letters (Parkinson, 1972), showed less forgetting if presented visually than if presented aurally when subjects shadowed aurally presented material during the retention interval. In addition, Kroll (1972) and Parkinson et al. (1971) found that shadowing phonemically similar material during the retention interval interfered with recall of a letter presented aurally, but not with one presented visually.

Hall, Swane, and Jenkins (1973) maintained that such short-term visual storage was based on the physical as opposed to the auditory-verbal-linguistic features of the input. They found that visually presented geometric figures were recalled better than the visually presented names of those figures when subjects were required to shadow unrelated words during the retention interval. Hall et al. (1973) argued that this provided evidence that physical properties are held in a nonverbal visual memory not subject in interference from auditory-verbal-linguistic memu $y$. The present study was designed to further investigate this hypothesis, especially in the light of Parkinson's (1972) finding that four letters can be held in a visual memory store not subject to interference from an auditory shadowing task. In particular, it is argued that if names of shapes presented visually áre transformed into an a uditory-verbal-linguistic code then the retention

Requests for reprints should be addressed to the first author. now at the School of Behavioural Sciences, Macquarie University. North Ryde, New South Wales, Australia 2113. characteristics of material should be similar to that of the same names presented aurally, at least over the range of retention intervals beyond the duration of echoic memory. This hypothesis was investigated in both experiments of the study.

In Experiment 1 different groups of subjects were presented with a single slide containing either geometric figures, the names of those figures. or were presented aurally with the figures' names. Subjects were then required to shadow aurally presented unrelated words during the retention interval. It was predicted. in line with the findings of Hall et al. (1973), that the geometric figures should show little, if any, retention loss while the two name groups should show similar marked retention losses over the retention intervals used.

Experiment II was a replication of Experiment I with the exception that the shadow task was presented visually. This was included to determine whether the visual storage was dependent critically on the interpolated task being auditory in nature. If "physical" storage is more efficient when subjects are required to "process" other materials in an auditory fashion then it might reasonably be asked what the effect would be of a limited amount of visual processing of other materials on "physically" stored material. The qualification "limited amount of visual processing" seems necessary since, although the intervening task in the experiment is visual in nature, a priori it would seem to require the same type of processing as in Experiment I, i.e., subjects are required to repeat words out loud as they see or hear them. On these grounds it was predicted that both tasks would interfere with memory material in a similar way.

\section{EXPERIMENT I}

Method

Forty-five first-y'ear undergraduate students in psychology at 


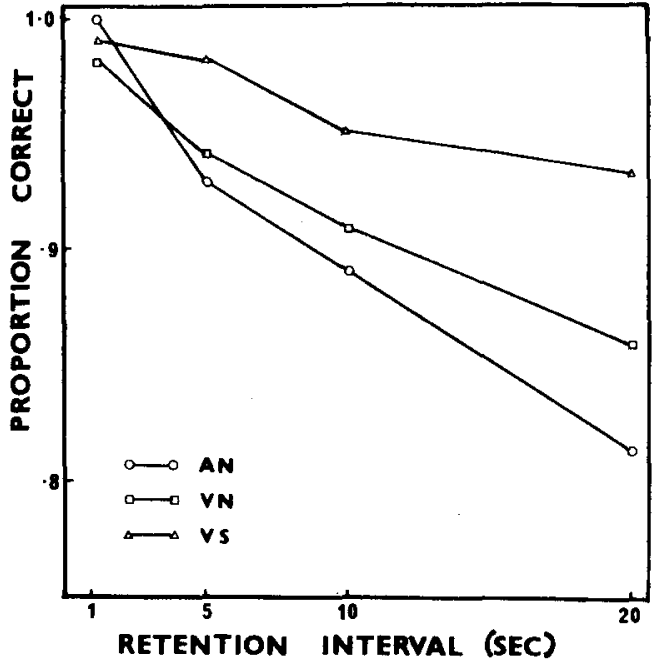

Figure 1. Proportion of correct recall responses over retention intervals for Experiment $I$.

the University of Sydney served as subjects, participation in experiments being part of the course requirement. They were assigned randomly to three groups, 15 subjects per group, with the groups differing in the nature of the to-be-remembered material. Group VS received slides of geometric figures; Group VN received slides of the names of those figures; and Group AN received the figure names aurally. The figures were constructed from four dimensions, the dimensions and values being: shape (square, circle, or triangle), number of figures (two or three), color (black or white), and size (large or small). Twenty-four slides were obtained by combining all the above attributes. A second set of 24 slides contained the four words necessary to name each of the figures displayed in the natural reading order, i.e., number, followed by size, followed by color, followed by form. This presentation order was the same for the auditory memory material. This was recorded in a male voice so as to clearly distinguish it from material to be shadowed, which was recorded in a female voice. The presentation time of the memory material was $1.5 \mathrm{sec}$ in all conditions based on the time needed for aural presentation for Group AN. A $1000-\mathrm{Hz}$ tone was sounded $2 \mathrm{sec}$ before presentation of memory material, again immediately prior to presentation, and also immediately following presentation.

Visual material was projected onto a screen on the wall of the laboratory by means of a manual slide projector controlled by a decade interval timer. Aural material was prerecorded on a Hanimex twin track tape recorder.

The words to be shadowed were two-syllable words chosen from Australian daily newspapers on the assumption that these words were more likely to conform to Australian norms and usage. They were presented at a rate of two words per second over stereo headphones to the seated subject.

Subjects first completed 10 practice trials to accustom them to the shadowing task. A single random order of the practice shadowed lists was used for all subjects. The first 4 of these involved shadowing only and consisted of 32-word lists. The next 6 practice trials included presentation of memory material aurally in Group AN and visually in groups VS and VN. The memory material consisted of single digits for the first 3 such practice trials and letters for the next 3 where the letters could vary in size and number. The subject was instructed to commence shadowing immediately following offset of the memory material and to be as accurate as possible in the shadowing task. The end of the shadowing list was marked by a $1000-\mathrm{Hz}$ tone, after which the subject was instructed to write down the memory material, guessing where necessary.
Following these practice trials, each subject received 24 experimental trials composed of 6 trials at each of four retention intervals, the intervals being $1,5,10$, and $20 \mathrm{sec}$. The order in which retention intervals were assigned to the 24 experimental trials was random, subject to the constraint that any one retention interval did not occur on more than 2 successive trials. A single random order of the shadowed lists and associated memory material was used for all subjects. The subjects were informed of the attribute-value composition of the memory material and, as in the practice trials, all subjects were required to write their responses on a slip of paper and hand it to the experimenter. All experimental trials were separated by a random time interval between 45 and $70 \mathrm{sec}$ to minimize build-up of proactive inhibition (Kincaid \& Wickens, 1970).

\section{Results}

To insure all groups had received an equivalent level of performance in shadowing, a one-way analysis of variance was carried out on the arcsine transformed proportions of words correctly shadowed over Trials 24 . No significant differences were found among the groups $(F=0.31, \mathrm{df}=2,42, \mathrm{p}>.05)$ which means that all groups were equated for shadowing performance. However, a significant difference was obtained on the experimental trials $(\mathrm{F}=14.9, \mathrm{df}=2,42, \mathrm{p}<.001)$. Post hoc Scheffe tests on the means revealed that the difference was due to poorer shadowing in Condition AN $(73 \%$ correct $)$ than in the other conditions ( $87 \%$ and $84 \%$ for conditions VN and VS, respectively).

Recall performance of each group is shown in Figure 1.

In counting the recall errors for the experimental trials each attribute was scored separately, so that a total of four errors was possible on each trial. A square root transformation of the number of errors at each of the longest three retention intervals was then subjected to a two-way analysis of variance. The shortest retention interval was deleted from this analysis due to the almost perfect recall in all groups. This interval was included simply to insure all memory material is equally perceived in the different memory modalities (Kroll et al.. 1970). The square root transformation $\left(x^{\prime}=\sqrt{x}+\sqrt{x+1}\right)$ was carried out as there was some tendency for cell means and variances to be correlated (Winer, 1971, P. 400). The results of this analysis showed that the main effect for retention intervals reached significance $(F=33.8$, $\mathrm{df}=1,42, \mathrm{p}<.001$ ) (standard error $=.82$ ), with recall showing a steady decline with increasing retention intervals for all groups. There was no significant interaction between groups across retention intervals $(F=0.54, d f=4,84, p>.05)$. Instead of testing the main effect for between groups, two planned contrasts were set up in order to test the predictions more directly. The first compared Groups VN and AN. No significant difference was obtained $(\mathrm{F}=2.5, \mathrm{df}=1,42$, $\mathrm{p}>.05$ ). The second compared Group VS with the other two Groups combined. Group VS showed better recall than Groups $\mathrm{VN}$ and $\mathrm{AN}(\mathrm{F}=22.8, \mathrm{df}=1,42$, $\mathrm{p}<.001)$. 


\section{Discussion}

The findings in this experiment support the hypothesis that visual short-term memory is based primarily on the physical properties of a visually presented stimulus as recall of figures was superior to recall of the names of those figures both when they were presented visually and aurally. The finding that recall of figure names was no different when presented visually than when presented aurally is consistent with the hypothesis that these are stored in the same manner. That both suffered interference from the shadowing task whereas the figures suffered very little interference suggests that both are stored in an auditory-verbal-linguistic code. This conclusion may, however, need to be revised in view of the fact that the shadowing performance of subjects in Group AN was poorer than that of subjects in Group VN. This point will be taken up further in the general discussion.

\section{EXPERIMENT II}

\section{Method}

Experiment II was the same in all respects as Experiment I with the exception that the shadowing task was visual in nature and there were fewer practice trials.

The visual material for shadowing was presented using a Telefunken magnetophon 300TS half-frame filmstrip projector which consists of a combined continuous projector and 2-track tape recorder unit. The projector and tape recorder could be synchronized by recording pulses on one track of the tape.

A continuous $35-\mathrm{mm}$ film strip was made up using the same words for shadowing material as in Experiment I. The presentation rate was three words/sec. It was found in a preliminary studty that subjects had no difficulty shadowing accurately at the two word $/ \mathrm{sec}$ rate used in auditory shadowing (Experiment I). The faster presentation rate for visual material gave approximately the same error rate as in Experiment $I$. The increased presentation rate necessitated the presentation of more words at each retention interval than in Experiment I. The same words were used in both experiments although in Experiment II some words were used twice.

Blank black frames were spliced into the film strip to separate the trials and allow presentation of a visual slide in Conditions VS and VN. The same set of slides was used in this experiment as in Experiment $\mathbf{I}$. Tones were also presented as in Experiment I.

A further 45 first-year undergraduate students in psychology served as subjects with 15 being randomly assigned to each of three groups: VS, VN, and AN as in Experiment I.

\section{Results}

No significant differences between groups were found in shadowing during practice trials $(\mathrm{F}=0.38, \mathrm{df}=2,42$, $\mathrm{p}>.05)$. nor during the experimental trials $(F=0.70$, $\mathrm{df}=2,42, \mathrm{p}>.05$ ). Thus, in contrast to Experiment $\mathrm{I}$, shadowing was not affected by presentation modality of the memory material.

Recall performance is shown in Figure 2. Analysis of variance of the square root transformed number of errors at each of the longest three retention intervals revealed a significant drop in recall across retention intervals $(F=i 1.2, d f=2,84, p<.001)$ (standard error $=.99)$, but no significant interaction between groups across retention intervals $(F=0.15, d f=4,84$, $\mathrm{p}>.05$ ). Groups $\mathrm{VN}$ and $\mathrm{AN}$ did not differ significantly, $(F=0.18, \quad d f=1,42, p>05)$, whereas Group VS showed better recall than Groups VN and AN combined $(F=13.96, d f=1,42, p<.001)$.

\section{Discussion}

The results of this experiment are consistent with those of Experiment I. Figures were recalled better than names, both when the latter were presented visually and aurally, and recall of names was not affected by modality of presentation of those names.

Visual shadowing was used in this experiment to test the hypothesis that visual short-term memory does not depend critically on intervening material being presented in modalities other than visual. The findings confirmed this hypothesis. That is, it is the act of repeating words which causes interference in the case of recall of the figure names, not the input modality.

Unlike Experiment I, analysis of shadowing performance on the experimental trials shows that presentation modality of memory material did not affect shadowing performance. It can thus be asserted more confidently in the present experiment that names of figures are stored in a similar manner, i.e. in an auditory-verbal-linguistic code.

\section{GENERAL DISCUSSION}

The findings in both experiments provide support for the hypothesis that visual short-term memory is based primarily on physical properties of the input. Four-word descriptions are apparently recoded into an auditory-verbal-linguistic code even when presented visually. This finding would seem at variance with

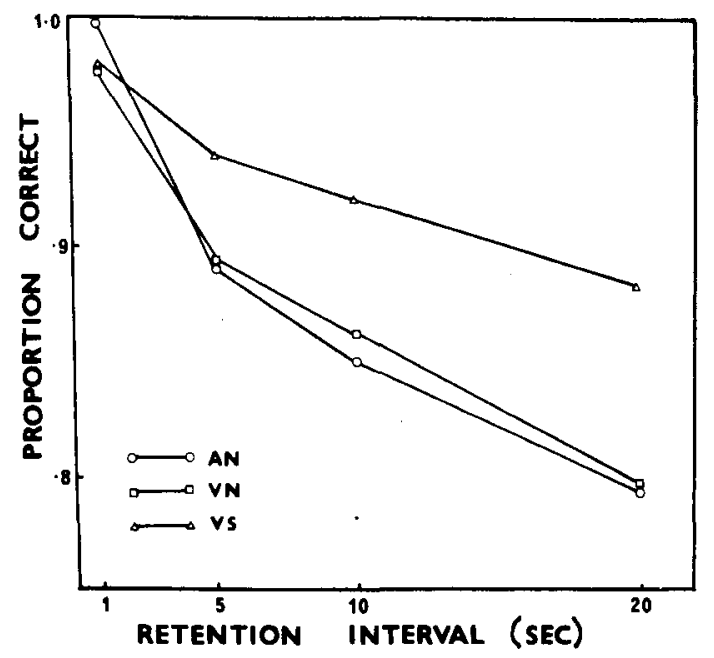

Figure 2. Proportion of correct recall responses over retention intervals for Experiment II. 
studies which have compared visual with auditory memory letters. The finding that four-word visual descriptions of figures are not retained as well as the actual figures is suggestive of the fact that visual short-term memory, as well as being based on physical properties, may be limited in capacity. That is, it may only be able to hold a limited amount of visual information at any one time. If this limited capacity hypothesis is true then it would help to explain discrepancies in previous findings.

Conrad (1964), Dale and Gregory (1966), and Sperling (1967), among others, have demonstrated that acoustic confusions occurred even when memory letters were presented visually. Typically, these experiments have presented six to eight items for recall. However, experimenters who claim to have demonstrated the existence of short-term visual memory have typically presented between one and four items for recall (e.g., Kroll et al., 1970; Parkinson, 1972; Scarborough, 1972) and have not found acoustic confusions. This difference would suggest that with memory letters the short-term memory system can hold a maximum of approximately five items. After this maximum has been passed items are recoded into an auditory or acoustic form and are thus likely to show acoustic confusion on recall. On this hypothesis it should also be possible to overload the memory capacity by presenting greater numbers of varieties of figures for recall.

One problem associated with postulating a visual short-term memory which is limited in capacity lies in accounting for the continuous visual recognition memory type studies (e.g., Kirsner, 1973) which suggest that many items can be held in visual memory. This possible anomaly may be tied to the paradigm used in the investigation of visual memory. Just how much this is the case is a matter for further empirical investigation.

The finding that visual short-term memory was unaffected by the modality of presentation of the shadowing material does not mean that interpolated visual material will not interfere with such memory. It is possible that material with similar physical properties to the material to be recalled will result in retention loss. This possibility seems worthy of investigation in view of the present findings regarding visual shadowing.

It seems a reasonable working hypothesis to suggest that when the same memory material is present in different ways, resulting in a similar recall decrement for the two types of material following an intervening task, that the decrement is due to the same kind of interference and/or storage. It is a suggestion, however, which should be regarded with some degree of caution, as evidenced by the shadowing performances in Experiment $\mathbf{I}$.

In Experiment I, although there was no significant difference in recall between Groups $\mathrm{AN}$ and VN, there was significant difference in shadowing performance between these two groups. Group VN shadowed significantly better than Group AN. The finding that shadowing performance was affected by presentation modality of the memory material in Experiment I could mean either that shadowing differences were unrelated to memory storage of the names or that aural presentation of the shadowing material caused some particular difficulty in storing the aurally presented memory material over and above the act of repeating the material. While the present study was not able unequivocally to distinguish between these two possibilities, or other interpretations, postexperimental protocols do provide some weak evidence for a possible interpretation. After the experiment, subjects were asked how they had retained the memory material. In Experiment I, where the presentation rate of shadowed material was two words per second, subjects' responses, independent of group condition, separated into two distinct categories; they either claimed to have "visualized" or to have "verbalized" the memory material. This breakdown showed that all subjects within a group who claimed to have "visualized" did better than all subjects who claimed to have "verbalized." However, in Experiment II, where the presentation rate of shadowed material was three words per second, no such group independent division of subjects' responses was noted. That is, all subjects in Group VS claimed to have "visualized," while all subjects in Groups VN and AN claimed to have "verbalized." This weak finding would seem to imply that in Experiment I, some subjects were able to further process the memory material to a more efficient type of storage while shadowing, resulting in a slight decrement in shadowing performance. Although this essentially subjective analysis does offer a partial solution to the problem of poorer shadowing in Experiment I for Group AN, if it is correct it would seem to raise a number of problems for studies using a similar paradigm to the present experiment, the most notable being the exact nature and effect of subjects' "control processes" given the different types of memory materials frequently used in conjunction with the different types of intervening tasks.

\section{REFERENCES}

Conrad, R. Acoustic confusion and immediate memory. Britich Joumal of Psy chology, 1964, 55, 75-84.

Dale, H. C. A., \& Gregory, M. Evidence of semantic coding in short-term memory. Psy chonomic Science, 1966, 5, 75-76.

Hall, R.. Swane, D. \& Jenkins, R. A. Memory for physical and semantic features of visual material in a shadowing task. Joumal of Experimental Psy chology, 1973, 100, 426-428.

Kincaid, J. P., \& Wickens, D. D. Temporal gradient of release from proactive inhibition. Joumal of Experimental Psy chology. 1970. 86, 313-316.

Kirsner, $K$. An analysis of the visual component in recogntion memory for verbal stimuli. Memory Cognition, 1979, 1 , $449-453$.

Kroll, N. E. Short-term memory and the nature of interference from concurrent shadowing. Quarterly Joumal of Experimental Psychology, 1972, 24, 414-419. 
Kroll, N. E., Parks, T., Parkinson, S. R., Bieber, S, L., \& Johnson, A. L. Short-term memory while shadowing: Recall of visually and aurally presented letters. Joumal of Experimental Psy chology, 1970, 85, 220-224.

Parkinson, S. R. Short-term memory while shadowing: Multiple item recall of visually and or aurally presented letters. Joumal of Experimental Psy chology, 1972, 92, 256-265.

Parkinson, S. R., Parks, T., \& Kroll, N. E. A. Visual and auditory short-term memory: The effects of phonemically simila auditory shadow material during the retention interval.Journal of Experimental Psy chology, 1971, 87, 274-280.
Scarborough, D. L. Stimulus modality effects on forgetting in short-term memory. Joumal of Experimental Psychology, $1972,95,285-289$

Sperling, G. Successive approximations to a model for short-term memory. Acta Psy chologica, 1967, 27, 285-29 2.

Winer, B. J. Statistical principles in experimental design, 2nd Ed. New York: McGraw-Hill, 1971.

(Received for publication October 18, 1974 Revision received March 17,1975 .) 